\title{
Primary squamous cell carcinoma of the esophagus initially presenting as a large retroperitoneal mass: A case diagnosed as cancer of unknown primary site
}

\author{
LANFANG YU $^{1}$, XIAOXIAO GE ${ }^{1}$, SUI HUANG $^{1}$, YANLI WANG $^{2}$ and PENG SHEN ${ }^{1}$ \\ Departments of ${ }^{1}$ Medical Oncology and ${ }^{2}$ Pathology, The First Affiliated Hospital, College \\ of Medicine, Zhejiang University, Hangzhou, Zhejiang 310000, P.R. China
}

Received September 11, 2012; Accepted January 30, 2013

DOI: $10.3892 / \mathrm{mco} .2013 .79$

\begin{abstract}
Retroperitoneal squamous cell carcinoma (SCC) of unknown origin is uncommon. It is extremely rare when the primary site detected in the esophagus after 18 months. A 59-year-old female patient with waist pain was initially diagnosed as retroperitoneal metastatic SCC of occult origin. Six cycles of chemotherapy with cisplatin, paclitaxel and 5-fluorouracil were administered and clinical complete response was observed. The primary site was detected in the esophagus after 18 months and the overall survival (OS) was 28 months. To the best of our knowledge, this is the first case of esophageal squamous cell carcinoma (ESCC) initially presenting as a metastatic site with long progression-free survival (PFS) and OS. In conclusion, the different biological characteristics and complete response to first-line chemotherapy likely contribute to relatively long PFS and OS.
\end{abstract}

\section{Introduction}

Squamous cell carcinoma (SCC) accounts for 5-10\% of diagnosed cancers of unknown primary (CUP) site (1). As reported, the primary site is detected in antemortem examinations in only $25 \%$ of patients (2) and median survival is predicted to be 6-9 months (3). A limited number of studies have described retroperitoneal CUP with primary site detected in the esophagus during follow-up, and even fewer studies have observed long progression-free survival (PFS) and overall survival (OS). In the present case study, we report a 59-year-old female patient with SCC presenting as large retroperitoneal mass with primary site in the esophagus detected subsequently.

Correspondence to: Professor Peng Shen, Department of Medical Oncology, The First Affiliated Hospital, College of Medicine, Zhejiang University, 79 Qingchun Road, Hangzhou, Zhejiang 310000, P.R. China

E-mail: zyhlk@sina.cn; shenp@zju.edu.cn

Key words: cancer of unknown primary, chemotherapy, esophageal cancer, squamous cell carcinoma
This patient was treated with chemotherapy, and had PFS of 18 months and OS of 28 months. Of note, a search of the literature using Medline, EMBASE and the Web of Science, until August 2012, yielded no similar reports.

\section{Case report}

A 59-year-old female patient was referred to a hospital with a 4-month history of persistent waist pain in August 2008. She was diagnosed with lumbar muscle strain. The patient ingested traditional Chinese medicine. However, the pain became more severe in the following three months and the patient was not able to lie back without strong opioid analgesics and lost $7 \mathrm{~kg}$ during this period of time. In November 2008, the patient was admitted to a second hospital. Physical examinations revealed nothing abnormal and the abdominal computed tomography (CT) scan showed that a large mass occupied the entire retroperitoneal cavity (Fig. 1). Positron emission tomography/computed tomography (PET/CT) showed a mass in the retroperitoneal cave, which was considered as lymph nodes blending together into a large mass, with an average standardized uptake value (SUV) of 5.5. Due to the limited conditions, the patient did not undergo biopsy and was diagnosed as having retroperitoneal occupation.

For further diagnosis and therapy, the patient was finally hospitalized at our Department on November 12th, 2008. Physical examination showed a mild tenderness in the middle of the upper abdomen. No other positive signs were found including palpable inguinal or cervical lymph nodes. Blood tests were normal other than an elevation of carcinoembryonic antigen (CEA) to $7.2 \mathrm{ng} / \mathrm{ml}$ (normal $\leq 5.0 \mathrm{ng} / \mathrm{ml}$ ) and carbohydrate antigen-199 (CA199) to $88.8 \mathrm{U} / \mathrm{ml}$ (normal $\leq 35.0 \mathrm{U} / \mathrm{ml}$ ). The abdominal CT showed a large mass with no clear boundaries fixed to the retroperitoneal cavity and enveloping the abdominal aorta as well as other vascular elements. No other lesions were detected by gastro-esophageal endoscopy, nasopharyngeal CT, chest CT or cervix and urology-gynecologic ultrasonography. An ultrasound guided puncture biopsy was conducted. The pathological examinations suggested proliferation of squamous cancer cells forming intercellular bridges and keratin pearls (Fig. 2). Immunohistochemical staining showed positive for cytokeratin (CK) 14 (Fig. 3) and p63 (Fig. 4), which 


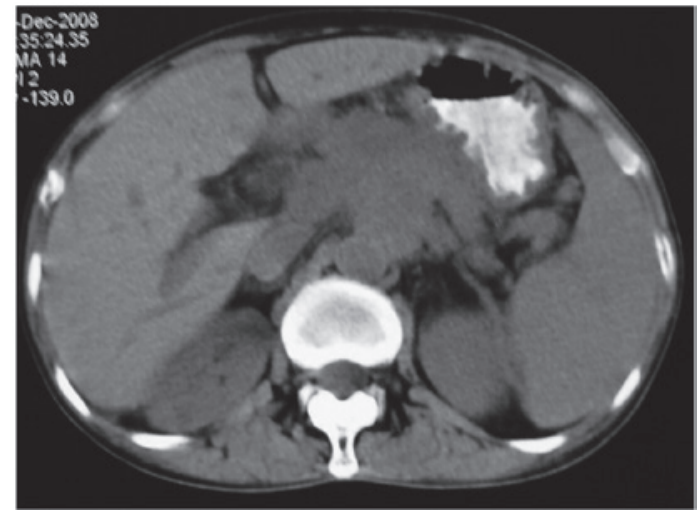

Figure 1. Abdominal computed tomography (CT) scan prior to chemotherapy showed a mass occupying the entire retroperitoneal cavity.

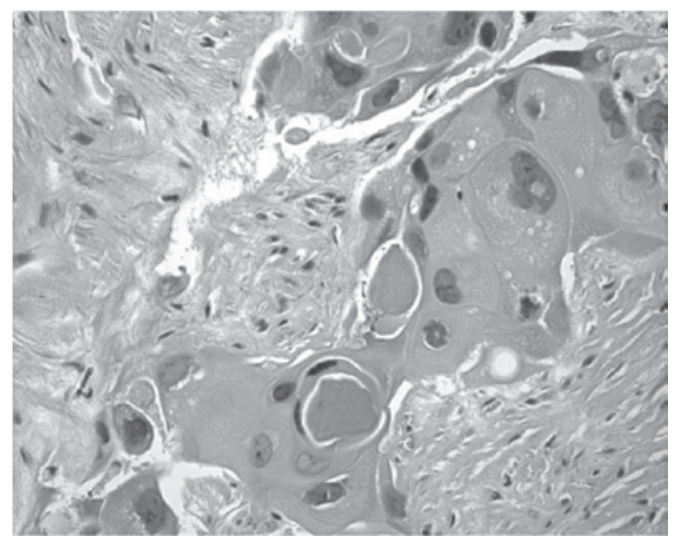

Figure 2. Hematoxylin and eosin staining (magnification, $\mathrm{x} 400$ ) revealed keratic pearl forming.

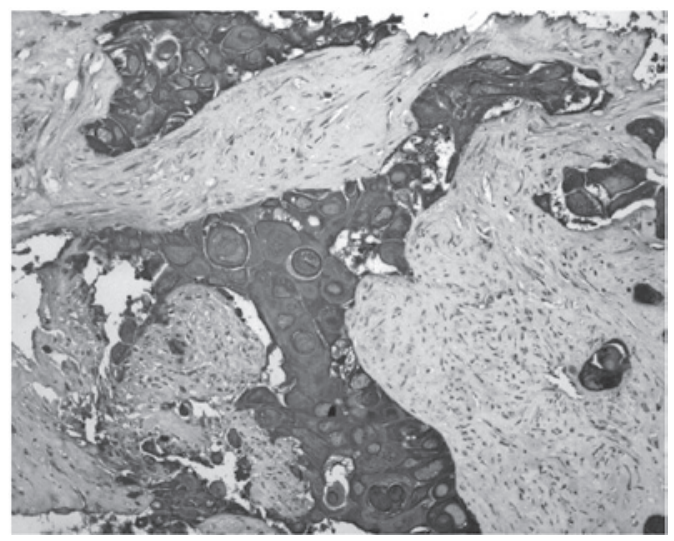

Figure 3. Immunohistochemical staining (magnification, x200) showed positive for CK14.

contributed to the diagnosis of SCC. Since no specific primary lesion was detected, the patient was ultimately diagnosed as retroperitoneal metastatic SCC of unknown primary site.

Subsequent to sound discussion among the multidisciplinary team (MDT), surgery and radiotherapy were not recommended at the time as the tumor was fixed to the entire retroperitoneal cavity, which had no clear boundaries

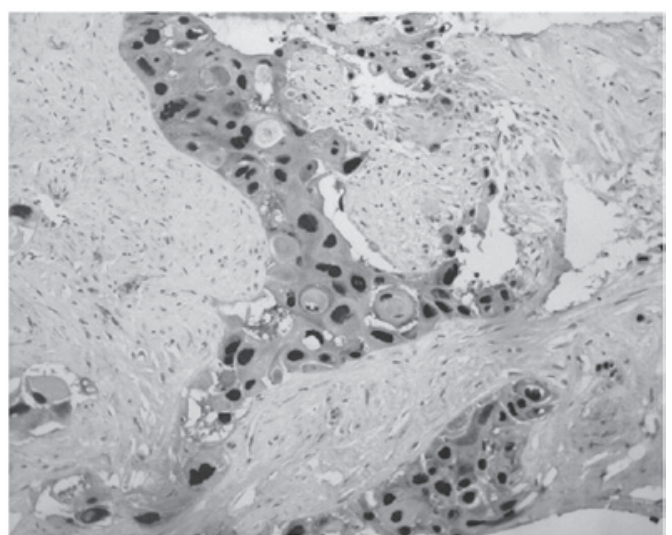

Figure 4. Immunohistochemical staining (magnification, $x 200$ ) showed positive for $\mathrm{p} 63$.

and enveloped the abdominal aorta as well as other vascular elements. Thus chemotherapy was selected as the first-line treatment. From December 19th 2008, the patient underwent six 21-day cycles of chemotherapy of cisplatin $\left(25 \mathrm{mg} / \mathrm{m}^{2} /\right.$ day on days 2-4), paclitaxel $\left(80 \mathrm{mg} / \mathrm{m}^{2} /\right.$ day on days 1 and 8$)$ and 5 -fluorouracil (500 mg/m²/day on days 1-3). After one cycle of chemotherapy, the waist pain was completely relieved. Serum levels of CEA and CA199 decreased to normal levels. At the end of the second cycle, clinical partial response of the lesion was confirmed by abdominal CT (Fig. 5) and clinical complete response (CCR) after six cycles (Fig. 6). Due to hematological toxicity paclitaxel was cancelled on day 8 in cycles 2 and 6 . Grade 3/4 toxicities (Common Toxicity Criteria, version 3.0) involving nausea/vomiting, neutropenia, hair loss and peripheral neurotoxicity were observed.

The patient was followed up for 18 months, during which she led a normal life. Periodical clinical and instrumental examinations showed no signs of tumor recurrence or metastasis until September 2010, when she was referred to a hospital, complaining of recent onset of dysphagia. Esophagogastroduodenoscopy showed a mass in the middle-low esophageal lumen. Biopsy of the mass was performed and histological examination revealed SCC. Following detailed examinations, no other lesions were found and the diagnosis was clarified as CUP with the subsequent primary site identified in the esophagus. Subsequent treatment strategies were discussed among the MDT. Surgery was not recommended as the metastatic site had already been identified. However, the patient was weak and could not tolerate the toxicity of the chemotherapy. Thus, she underwent radiotherapy of the tumor and the regional lymph nodes. Following radiotherapy, stable disease was confirmed. The patient was kept free of disease progression for 3 months. In March 2011, the patient was referred to hospital complaining of more severe dysphagia. No chemotherapy or radiotherapy was considered due to her condition. Supportive care was provided, and the patient succumbed to the disease one month later, with OS of 28 months.

\section{Discussion}

CUP is defined as histologically proven metastatic malignant tumor whose primary site cannot be identified during 
Table I. PFS and OS in advanced ESCC after first-line chemotherapy.

\begin{tabular}{lllcccrrr}
\hline Authors (Refs.) & Date & Country & Total sample size & Size of SCC & \multicolumn{2}{c}{ Chemotherapy drugs } & PFS \\
\hline Cao (11) & 2009 & China & 48 & 46 & Nedaplatin, paclitaxel & $6.1 \mathrm{~m}$ & $11.5 \mathrm{~m}$ \\
Shim (12) & 2010 & Korea & 38 & 38 & Cisplatin, 5-fluorouracil & $7.5 \mathrm{~m}$ & $7.4 \mathrm{~m}$ \\
Honda (13) & 2010 & Japan & 41 & 41 & Doxirubicin, cisplatin, 5-fluorouracil & $153 \mathrm{~d}$ & $306 \mathrm{~d}$ \\
Kim (14) & 2010 & Korea & 39 & 39 & Docetaxel, cisplatin & $5 \mathrm{~m}$ & $8.3 \mathrm{~m}$ \\
Overman (15) & 2010 & USA & 117 & 17 & Docetaxel, cisplatin, 5-fluorouracil & $5.3 \mathrm{~m}$ & $8.9 \mathrm{~m}$ \\
\hline
\end{tabular}

PFS, progression-free survival; OS, overall survival; ESCC, esophageal squamous cell carcinoma; SCC, squamous cell carcinoma; m, months; d, days.

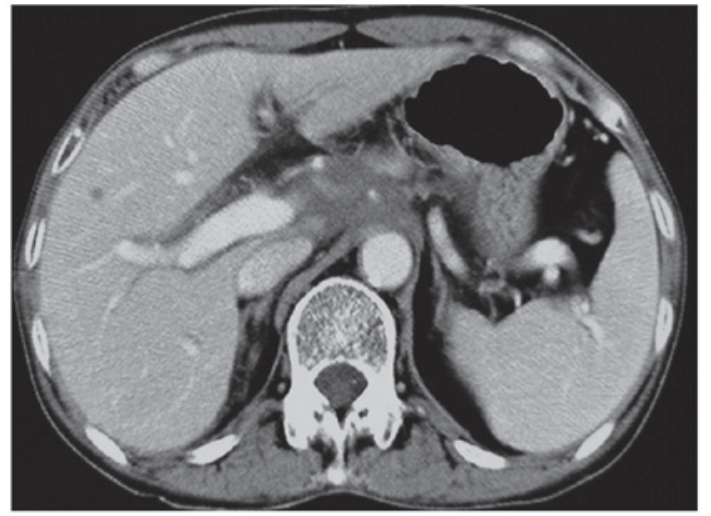

Figure 5. Abdominal computed tomography (CT) scan after two cycles of chemotherapy showed clinical partial regression.

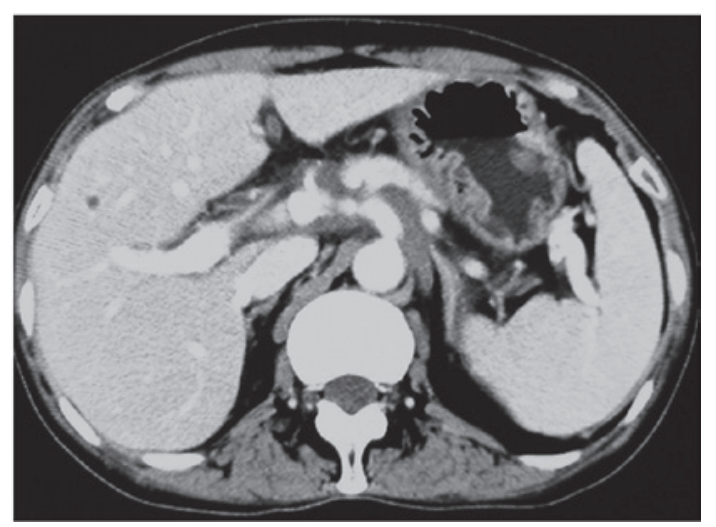

Figure 6. Abdominal computed tomography (CT) scan after six cycles of chemotherapy showed clinical complete regression.

pretreatment evaluation, accounting for 5-10\% of the malignancies diagnosed (4). There are four major types of CUP and SCC comprising 5-10\% of malignancies compared to 55-60\% for adenocarcinoma (ADC) (1). As previously reported, in only $25 \%$ of patients the primary site is detected in antemortem examinations (2). A previous study with almost 1,000 patients revealed that lung and pancreatic cancers were the most frequent primary sites identified (almost half of all cases), although esophageal cancer was not observed (5). Numerous sites of involvement are observed in $26 \%$ of CUP patients, while the most common isolated sites are liver (24\%) and lung (12\%), followed by lymph nodes (11\%) (6). Esophageal primary cancer also presents with lymph node involvement, with cervical lymph nodes $(30-49.5 \%)$ being more common than abdominal ones $(8-12 \%)(7,8)$. Thus, it is rare to observe squamous CUP presenting with solitary retroperitoneal lymph node metastasis. Detection of the primary site in the esophagus 18 months later is extremely rare. In fact, a search of published studies on Medline, EMBASE and the Web of Science, until October 2011, yielded no similar studies to the present one.

Prognosis of CUP is poor and median survival is predicted to be 6-9 months (3). Previous studies discussed multidisciplinary treatment of advanced esophageal carcinoma, which showed that palliative resection was not recommended and induction chemotherapy followed by chemoradiotherapy (CRT) was feasible $(9,10)$. Neither of these studies focused on the treatment of retroperitoneal lymph node metastasis of SCC. Since the mass had no clear boundaries and enveloped important vascular elements, surgery and radiotherapy were not recommended. Thus, a platinum-based chemotherapy regimen was chosen according to the National Comprehensive Cancer Network (NCCN 2008 version 1.1) guidelines. Moreover, the PFS and OS in advanced esophageal squamous cell carcinoma (ESCC) following first-line chemotherapy were not satisfied, with ranges of 4.5-6.1 and 7.4-11.5 months, respectively (Table I) (11-15). Compared with patients in those studies, the outcome of the patient in our case was improved, with PFS of 18 months and OS of 28 months.

Therefore, it is likely that the different biological characteristics resulting in a different outcome between carcinomas with reverse sequence of the primary site and metastatic site played a role. Findings of an early study (16) showed that the percentage of the osseous involvement was much lower in lung cancer presenting as CUP than that in known primary cancers, which was opposite to findings in pancreatic and prostatic cancers (16). However, investigations of esophageal cancers in the studies searched were not identified. Thus, more relevant studies investigating the role of the biological characteristics in the prognosis of such cancers should be conducted.

The hypothesis of whether a complete response following first-line therapy is beneficial to OS was considered. Keskin et al (17) reported findings on 410 patients with locally advanced breast cancer who had undergone surgery following neoadjuvant chemotherapy. A longer PFS was noted in patients achieving pathological complete response 
(pCR) after neoadjuvant chemotherapy, although no increase in OS was observed. Results from the TECHNO trial of the AGO and GBG study groups revealed that among the patients with human epidermal growth factor receptor 2 (HER-2)-overexpressing breast cancer, $\mathrm{pCR}$ after neoadjuvant among anti-HER2 therapy in combination with chemotherapy followed by maintenance trastuzumab had an improved long-term outcome (18). Apart from the aforementioned studies on breast cancer patients who received preoperative chemotherapy, a previous study (19) reported findings on 31 patients of esophageal cancer with distant lymph node metastasis. Only four of these 19 patients survived for $>3$ years and half of the survivors were those who showed CCR after definitive CRT (19). In their study, Yamamoto et al (20) reported a patient of advanced ESCC treated with chemotherapy with docetaxel, 5-fluorouracil and cisplatin. CCR was confirmed after 2 cycles of chemotherapy and OS of 4 years was observed. However, no large-scale studies on palliative chemotherapy as mentioned above were identified, thus, more clinical studies regarding this mechanism are required.

In summary, to the best of our knowledge, this is the first case of esophageal SCC initially presenting as metastatic site of a large retroperitoneal mass. Clinical complete response was confirmed following first-line chemotherapy. PFS of 18 months and OS of 28 months were observed. Thus, the different biological characteristics and/or complete response to chemotherapy may contribute to the relatively long PFS and OS. Nevertheless, large-scale studies are required to determine the mechanism of action.

\section{References}

1. Oien KA: Pathologic evaluation of unknown primary cancer. Semin Oncol 36: 8-37, 2009.

2. Hillen HF: Unknown primary tumours. Postgrad Med J 76: 690-693, 2000.

3. Pavlidis N, Briasoulis E, Hainsworth J and Greco FA: Diagnostic and therapeutic management of cancer of an unknown primary. Eur J Cancer 39: 1990-2005, 2003.

4. Greco FA and Hainsworth JD: Tumors of unknown origin. CA Cancer J Clin 42: 96-115, 1992.

5. Pentheroudakis G, Golfinopoulos V and Pavlidis N: Switching benchmarks in cancer of unknown primary: from autopsy to microarray. Eur J Cancer 43: 2026-2036, 2007.

6. van de Wouw AJ, Janssen-Heijnen ML, Coebergh JW and Hillen HF: Epidemiology of unknown primary tumours; incidence and population-based survival of 1285 patients in Southeast Netherlands, 1984-1992. Eur J Cancer 38: 409-413, 2002.
7. Akiyama H, Tsurumaru M, Udagawa $H$ and Kajiyama Y: Radical lymph node dissection for cancer of the thoracic esophagus. Ann Surg 220: 364-373, 1994.

8. Chen J, Liu S, Pan J, et al: The pattern and prevalence of lymphatic spread in thoracic oesophageal squamous cell carcinoma. Eur J Cardiothorac Surg 36: 480-486, 2009.

9. Kelsen DP, Winter KA, Gunderson LL, et al: Long-term results of RTOG trial 8911 (USA Intergroup 113): a random assignment trial comparison of chemotherapy followed by surgery compared with surgery alone for esophageal cancer. J Clin Oncol 25: 3719-3725, 2007.

10. Ruhstaller T, Templeton A, Ribi K, et al: Intense therapy in patients with locally advanced esophageal cancer beyond hope for surgical cure: a prospective, multicenter phase II trial of the Swiss Group for Clinical Cancer Research (SAKK 76/02). Onkologie 33: 222-228, 2010.

11. Cao W, Xu C, Lou G, et al: A phase II study of paclitaxel and nedaplatin as first-line chemotherapy in patients with advanced esophageal cancer. Jpn J Clin Oncol 39: 582-587, 2009.

12. Shim HJ, Cho SH, Hwang JE, et al: Phase II study of docetaxel and cisplatin chemotherapy in 5-fluorouracil/cisplatin pretreated esophageal cancer. Am J Clin Oncol 33: 624-628, 2010.

13. Honda M, Miura A, Izumi Y, et al: Doxorubicin, cisplatin, and fluorouracil combination therapy for metastatic esophageal SCC. Dis Esophagus 23: 641-645, 2010.

14. Kim JY, Do YR, Park KU, et al: A multi-center phase II study of docetaxel plus cisplatin as first-line therapy in patients with metastatic squamous cell esophageal cancer. Cancer Chemother Pharmacol 66: 31-36, 2010.

15. Overman MJ, Kazmi SM, Jhamb J, et al: Weekly docetaxel, cisplatin, and 5-fluorouracil as initial therapy for patients with advanced gastric and esophageal cancer. Cancer 116: 1446-1453, 2010.

16. Nystrom JS, Weiner JM, Heffelfinger-Juttner J, Irwin LE, Bateman JR and Wolf RM: Metastatic and histologic presentations in unknown primary cancer. Semin Oncol 4: 53-58, 1977.

17. Keskin S, Muslumanoglu M, Saip P, et al: Clinical and pathological features of breast cancer associated with the pathological complete response to anthracycline-based neoadjuvant chemotherapy. Oncology 81: 30-38, 2011.

18. Untch M, Fasching PA, Konecny GE, et al: Pathologic complete response after neoadjuvant chemotherapy plus trastuzumab predicts favorable survival in human epidermal growth factor receptor 2-overexpressing breast cancer: results from the TECHNO trial of the AGO and GBG study groups. J Clin Oncol 29: 3351-3357, 2011

19. Okamura S, Fujiwara H, Shiozaki A, et al: Long-term survivors of esophageal carcinoma with distant lymph node metastasis. Hepatogastroenterology 58: 421-425, 2011.

20. Yamamoto Y, Ikeda M, Takashima M, et al: A case of a patient with stage IVa esophageal cancer surviving over 4 years by combination chemotherapy with docetaxel, 5-FU and cisplatin, without operation. Gan To Kagaku Ryoho 38: 1321-1324, 2011 (In Japanese). 